Methods Quality and Outcomes Framework (QOF) electronic records until May 2016 were linked to mortality data. COPD Prevalence was calculated by sex, age group (10-year age bands), and SES using SIMD quintiles. Smoking status (ever smoked and current smoker) was also collected by the QOF. Population estimates for smoking status by age sex and SIMD for GGC were calculated using three Scottish Household Survey rounds, 2013, 2014 and 2015. COPD prevalence rates by SIMD quintile were calculated, adjusting for age, sex, and smoking status.

Results Crude prevalence of COPD among all ages in GGC is $2.74 \%$ and among those aged 40 years + in GGC was $5.67 \%$, higher in females $5.95 \%$ than males, $5.36 \%$. Comparing prevalence of COPD between males and females, rates were higher for males until age 39 and equivalent for ages 40-49 years. However, for 50-59 year olds prevalence among females was 3.84 compared with 3.15 among males, and for 60-69 year olds, prevalence was $8.15 \%$ for females compared with $7.26 \%$ for males. Thereafter prevalence was greater among males; for $70-79,80-89$ and $90+$ years, prevalence among males was $11.81 \%, 12.03 \%$ and $7.56 \%$ respectively, compared with $11.76 \%, 10.58 \%$ and $6.38 \%$ among females. Prevalence of COPD in SIMD 1 (most deprived) was almost 3.5 times of that in SIMD 5 (least deprived). Adjusting for age and sex, SES inequalities in COPD increased with SIMD1 prevalence 4.8 times that of SIMD5. After adjustment for age sex and ever smoked, SIMD1 prevalence was 3.1 times that of SIMD5. After adjustment for age, sex and current smoking, SIMD1 prevalence was 2.45 times that of SIMD5.

Conclusion Prevalence of COPD in GGC is higher than previously estimated. It is also higher among females than males at ages 50-70 years. Inequalities in COPD are evident and become greater on adjustment for age and sex. Smoking accounts for around half of the gap in prevalence of COPD between most and least deprived, however inequalities in COPD persist after adjustment for smoking status.

\section{RF8 LIFE COURSE SOCIOECONOMIC CIRCUMSTANCES AND DEMENTIA PREVALENCE: EVIDENCE FROM THE ENGLISH LONGITUDINAL STUDY OF AGEING}

${ }^{1} \mathrm{D}$ Cadar*, ${ }^{2} \mathrm{~L}$ Wright, ${ }^{2} \mathrm{P}$ Zaninotto. ${ }^{1}$ Department of Behavioural Science and Health, University College London, London, UK; ${ }^{2}$ Department of Epidemiology and Public Health, University College London, London, UK

\subsection{6/jech-2018-SSMabstracts.97}

Background Dementia represents a major public health impact. Previous work showed that higher socio-economic status (SES) is protective against dementia, through mentally engaging and socially interactive occupations and activities. However, the associations with childhood SES and social mobility are not well understood. We examined four SES indicators (father's social class, own education, occupational class and wealth) as well as social mobility across life, in relation to dementia prevalence.

Methods The data used are from 2032 men and women aged $\geq 65$ years at recruitment, from the English Longitudinal Study of Ageing (ELSA), an ongoing, representative prospective cohort study. Seven waves of data between 2002/03 (wave 1) and 2014/15 (wave 7) were analysed. Dementia was determined by doctor-diagnosis combined with a score above the threshold of 3.38 on the Informant Questionnaire on Cognitive Decline in the Elderly. Education has been grouped into university degree, A-levels and no education, and occupation into professional, intermediate, routine and manual. Wealth (property, savings, or other financial assets) was divided in quintiles, while social mobility was derived by combining childhood SES and adult occupational class into three stable SES levels (low, medium, high), and upwards or downwards trends. The highest SES indicator or stable-high were used as reference groups. Multivariable logistic regressions were employed to estimate the associations between each baseline SES indicator and dementia prevalence by wave 7 , while controlling for age, sex, marital status, long-standing limiting illness, and subsequent gradual adjustment for all other SES markers.

Results During the 12-year follow-up, $25 \%$ of sample developed dementia. Lower childhood SES was associated with a higher dementia risk (Odds Ratio (OR) $=1.39$ (95\% Confidence Intervals (CI) 1.02-1.89), but explained by education. Education did not show a protective effect, but the lower occupational class was associated with higher dementia risk $(\mathrm{OR}=1.39(95 \% \mathrm{CI}) 1.02$ to 1.89$)$. However, this association was subsequently explained by wealth. Lowest wealth was a strong predictor of dementia, independent of other SES markers (OR=2.81 (95\% CI) 1.83 to 4.32$)$ in contrast to the wealthiest counterparts. Those in the stable-low category across life showed a higher risk $(\mathrm{OR}=1.65(95 \% \mathrm{CI}) 1.01$ to 2.68) in contrast with those in stable-high SES. Upward or downward SES mobility trends did not show an impact.

Conclusion In an English, nationally representative sample, the incidence of dementia appeared to be socioeconomically patterned, primarily by the level of wealth and long-term SES disadvantage. Public health strategies for dementia prevention should target the socioeconomic gap to reduce health disparities and protect those who are particularly disadvantaged.

\section{RF9 DOES MOVING INTO SOCIAL, INTERMEDIATE AND MARKET-RENT ACCOMMODATION IN EAST VILLAGE (THE FORMER LONDON 2012 OLYMPIC ATHLETES VILLAGE) IMPROVE SELF-RATED MENTAL HEALTH, WELL-BEING AND NEIGHBOURHOOD PERCEPTIONS? EVALUATION OF A NATURAL EXPERIMENT}

${ }^{1} B$ Ram*, ${ }^{1} A R$ Rudnicka, ${ }^{1} \mathrm{~A}$ Shankar, ${ }^{1} \mathrm{CM}$ Nightingale, ${ }^{1} \mathrm{ES}$ Limb, ${ }^{2} \mathrm{~S}$ Cummins, ${ }^{2} \mathrm{D}$ Lewis, ${ }^{3}$ BG Corti, ${ }^{4}$ A Ellaway, ${ }^{5}$ AS Cooper, ${ }^{5} \mathrm{~A}$ Page, ${ }^{1} \mathrm{PH}$ Whincup, ${ }^{1} \mathrm{DG}$ Cook, ${ }^{1} \mathrm{CG}$ Owen. ${ }^{1}$ Population Health Research Institute, St. George's, University of London, London, UK; ${ }^{2}$ Department of Social and Environmental Health Research, London School of Hygiene and Tropical Medicine, London, UK; ${ }^{3}$ Enabling Capability Platforms, RMIT University, Melbourne, Australia; ${ }^{4}$ MRC/CSO Social and Public Health Sciences Unit, University of Glasgow, Glasgow, UK; ${ }^{5}$ Centre for Exercise, Nutrition and Health Sciences, University of Bristol, Bristol, UK

\subsection{6/jech-2018-SSMabstracts.98}

Background Evidence suggests that where we live might be an important determinant of mental health and well-being, especially amongst the more disadvantaged. However, longitudinal evidence is limited. The Examining Neighbourhood Activities in Built Living Environments in London (ENABLE London) study aimed to establish whether mental health, well-being, and neighbourhood perceptions improved among adults relocating to East Village, purposely designed for healthy active living, when compared with a control population who lived outside East Village throughout. 